\title{
Role of long non-coding RNA H19 in the development of osteoporosis
}

\author{
Senxiang Chen, Da Liu* (1), Zimo Zhou and Sen Qin
}

\begin{abstract}
Background: Osteoporosis is a widespread and serious metabolic bone disease. At present, revealing the molecular mechanisms of osteoporosis and developing effective prevention and treatment methods are of great significance to health worldwide. LncRNA is a non-coding RNA peptide chain with more than 200 nucleotides. Researchers have identified many IncRNAs implicated in the development of diseases and IncRNA H19 is an example.

Results: A large amount of evidence supports the fact that long non-coding RNA (IncRNA) genes, such as H19, have multiple, far-reaching effects on various biological functions. It has been found that IncRNA H19 has a role in the regulation of different types of cells in the body including the osteoblasts, osteocytes, and osteoclasts found in bones. Therefore, it can be postulated that IncRNA H19 affects the incidence and development of osteoporosis.

Conclusion: The prospect of targeting IncRNA H19 in the treatment of osteoporosis is promising because of the effects that IncRNA H19 has on the process of osteogenic differentiation. In this review, we summarize the molecular pathways and mechanisms of IncRNA H19 in the pathogenesis of osteoporosis and summarize the research progress of targeting $\mathrm{H} 19$ as a treatment option. Research is emerging that explores more effective treatment possibilities for bone metabolism diseases using molecular targets.
\end{abstract}

Keywords: Long noncoding RNA, IncRNA H19, Osteogenesis, Osteoporosis

\section{Background}

Osteoporosis is a widespread metabolic bone disease and the risk of developing it increases with age (Zou et al. 2020). The main characteristics of this debilitating disease are bone loss, bone density reduction, bone fragility, and the risk of fractures (Rachner et al. 2011). Because of problems with underdiagnosis, it is difficult to determine the variation in the incidence and prevalence of osteoporosis worldwide. Therefore, the best way to compare osteoporosis among different population groups is to observe the fracture rates (Johnston and Dagar 2020). Worldwide, osteoporosis causes more than 8.9 million fractures annually, resulting in an osteoporotic fracture every $3 \mathrm{~s}$ (Noh et al. 2020). As life expectancy is increasing

*Correspondence: spinecmu@163.com

Department of Orthopedics, Shengjing Hospital of China Medical

University, Shenyang 110004, Liaoning, China globally, osteoporosis will cause a severe economic burden in most countries. Osteoporosis-related fractures cost approximately $\$ 17.9$ and $£ 4$ billion per annum in the USA and the UK, respectively (Hernlund et al. 2013). And it is estimated that the worldwide economic cost of osteoporosis is expected to increase to $\$ 131.5$ billion by 2050 (Harvey et al. 2010).

The occurrence of osteoporosis is related to many genetic and environmental factors. These factors mainly affect the incidence and development of osteoporosis by regulating the activity and differentiation of osteoblasts and osteoclasts (Trajanoska and Rivadeneira 2019). Human bone metabolism includes bone formation by osteoblasts and bone resorption by osteoclasts. There are existing drugs to treat osteoporosis that inhibit the activity of osteoclasts. These include anti-bone resorption drugs such as bisphosphonates and the receptor activator of nuclear factor-kb ligand (RANKL) inhibitor, original author(s) and the source, provide a link to the Creative Commons licence, and indicate if changes were made. The images or other third party material in this article are included in the article's Creative Commons licence, unless indicated otherwise in a credit line to the material. If material is not included in the article's Creative Commons licence and your intended use is not permitted by statutory regulation or exceeds the permitted use, you will need to obtain permission directly from the copyright holder. To view a copy of this licence, visit http://creativecommons.org/licenses/by/4.0/. 
denosumab, which inhibits osteoclast formation, function, and survival. These drugs, however, have complicated side effects, and there is currently no optimal drug treatment for osteoporosis (Khan et al. 2017). Consequently, the exploration of targets for anabolic therapy, or the stimulation of bone formation by pharmacologic means, is an important emerging research focus. Understanding the molecular mechanisms of osteoporosis and developing effective prevention and treatment methods targeted to these mechanisms are of great importance to the health of an aging world population.

Recently, epigenetic studies have provided new insights into the treatment of osteoporosis. Epigenetic changes affect gene expression without changing the DNA sequence (Yang et al. 2020) and include DNA methylation, histone modification, and RNA-based mechanisms (van Wijnen and Westendorf 2019). With recent research developments, accumulating data suggest that lncRNA plays an important role in regulating bone cells and, therefore, affects the occurrence and development of osteoporosis (Hassan et al. 2015).

LncRNA is a non-coding RNA peptide chain with more than 200 bases and does not translate protein. Previously regarded as translation noise, lncRNA is now a prime focus of study in the rapidly developing field of genomics (Schmitz et al. 2016). Researchers have identified many lncRNAs implicated in the development of diseases and lncRNA $H 19$ is an example. For instance, lncRNA H19 regulates multiple myeloma by targeting miRNA-152-3p (Zheng et al. 2020) and is also a therapeutic target for pancreatic cancer (Sun et al. 2019). However, the process of how lncRNA $H 19$ modulates the pathogenesis of osteoporosis remains unclear. In this review, we discuss the role of lncRNA H19 in osteogenic differentiation, and summarize the relevant mechanisms of action to provide a theoretical basis for exploring lncRNA as a new molecular target in the treatment of osteoporosis.

\section{Discussion}

\section{Sources and classification of IncRNAs}

With the advancement of gene sequencing technology, a large number of non-coding lncRNAs have been discovered. LncRNA is a highly structured RNA transcription product. However, owing to the lack of an open reading frame, it does not translate protein and directly regulates gene expression at the RNA level (Jathar et al. 2017). It is also widely present in the cytoplasm and nucleus after transcription (Ponting et al. 2009). Generally, lncRNAs do not have coding potential, but some lncRNAs can code short peptides (Rion and Rüegg 2017). LncRNA is mostly transcribed by RNA polymerase II and most lncRNA transcripts have $5^{\prime}$-methylated caps and $3^{\prime}$-polyadenylic acid tails (Wu et al. 2013). However, some
lncRNAs are cleaved by the microprocessor to terminate transcription, or are bidirectionally transcribed, resulting in lncRNAs without $5^{\prime}$-methylated caps and $3^{\prime}$-end poly tails (Chen 2016).

LncRNAs can originate in various ways (Ponting et al. 2009) such as, by the disruption of the translation reading frame of protein-coding genes, from chromosomal reorganization, by non-coding genes through reverse transcription, and by the generation of non-coding RNA containing adjacent repeats through a partial tandem replication mechanism (Wei et al. 2017). LncRNA is highly involved in a variety of biological functions, but compared to the research of protein-coding sequences and micro-RNA (miRNA), lncRNA research is still early in its development. Extensive studies have been conducted on miRNA, and researchers have found that it can completely or incompletely base pair with target genes to regulate gene expression ( $\mathrm{Pu}$ et al. 2019). In comparison, lncRNA has a longer molecular sequence, a spatial structure, and a variety of modes of action, all of which allow for the possibility of many exploratory research pathways (Krol et al. 2015). The secondary structure, splicing form, and subcellular location of most lncRNAs are relatively conservative. This is particularly important for lncRNAs to function (Kopp and Mendell 2018). LncRNA can be divided into five categories according to their positions and genome sequences: sense, antisense, bidirectional, intronic, and intergenic (Ma et al. 2013; Wu et al. 2018a, b). In addition, IncRNAs can be divided into bait molecules, signal molecules, primers, and framework molecules according to molecular mechanisms (Wang and Chang 2011). These different types of lncRNAs perform a wide variety of functions.

\section{Common functions of IncRNAs}

LncRNAs have time and space specificity. The expression of lncRNAs varies in different tissues and the expression of IncRNA within the same tissue can also differ (Kim and Sung 2012). Because of these characteristics of lncRNA, its mechanism of action is complicated. Presently, a measure of the extent of all the actions of lncRNAs has not been fully elucidated, but there are roughly four types according to current research (Peng et al. 2018).

One type of lncRNA mechanism of action involves the regulation of epigenetic modification. Certain lncRNAs recruit chromatin remodeling and modification complexes to specific sites (Fang and Fullwood 2016). This changes the DNA/RNA methylation status, chromatin structure, and modification status. As a result, downstream gene expression is regulated by mediating chromatin remodeling or histone modification. For instance, hypomethylation of the promoter region of IncRNA SOX21-AS1 can make it overexpress, thereby enhancing 
the inhibitory effect of lncRNA SOX21 on lncRNA SOX2 and inhibit the progression of cervical cancer (Wang et al. 2019).

Another function of lncRNA is the regulation of transcriptional expression. In eukaryotic cells, transcription factors are vital for gene transcription. They can bind to the RNA produced by gene transcription to control RNA transcription, localization, and stability. Some lncRNAs act as ligands and bind to transcription factors to form a complex to control gene transcription activity by affecting the promoter region or adjacent genes (Dykes and Emanueli 2017). For instance, IncRNA GATA3-AS1 is a divergent lncRNA adjacent to IncRNA GATA3 and the GATA3-AS1 transcript alone is sufficient to induce an increase in GATA3 expression (Gibbons et al. 2018).

Dictating post-transcriptional regulation is an additional process in which lncRNA is involved. LncRNA participates in processes such as shearing, editing, protein translation, and transcription after mRNA transcription. By forming complementary double strands with mRNA, it interferes with the shearing of mRNA to produce a regulatory effect (Ransohoff et al. 2018).

The fourth type of IncRNA action is to function as competing endogenous RNA (ceRNA) to bind miRNA like a sponge, thereby preventing miRNA from binding to target mRNA. LncRNA also forms complementary duplexes with mRNA and small interfering RNA (siRNA) to regulate gene expression (Chan and Tay 2018).

\section{Mechanisms of action of IncRNAs in bone development}

Adult bone mass is maintained in homeostasis. To accomplish this, new bone formation replaces old bone tissue so that bone mass maintains a dynamic balance (Zhou et al. 2021). Osteoblasts, osteoclasts, and osteocytes are the main cells involved in this bone remodeling process. Osteoblasts are the main functional cells in the process of bone tissue formation. They are responsible for the synthesis, secretion, and mineralization of the bone matrix and are mainly differentiated from bone mesenchymal stem cells in the periosteum and bone marrow (Chen et al. 2015). Mesenchymal stem cells (MSCs) are pluripotent cells that can differentiate into osteoblasts, adipocytes or chondrocytes, and have become the preferred source of osteoporosis-based cell therapy. A variety of signaling pathways are involved in bone formation to regulate bone homeostasis, such as the $\mathrm{Wnt} / \beta$-catenin pathway, Notch pathway, and MAPK pathway (Majidinia et al. 2018). After the Wnt/ $\beta$-catenin pathway is activated, glycogen synthase 3 activity is reduced and $\beta$-catenin phosphorylation and proteasome degradation are inhibited. The stabilized $\beta$-catenin enters the nucleus to activate gene transcription and induce the proliferation and differentiation of osteoblasts (Nusse 2012). LncRNA regulates bone formation by affecting these signaling pathways. LncRNA $A N C R$ affects the Wnt/B-catenin pathway and inhibits osteogenic differentiation (Jia et al. 2015), while lncRNA HOTAIR affects the Wnt/ $\beta$-catenin pathway and inhibits osteogenic differentiation (Shen et al. 2019). The MAPK signaling pathways JNK, ERK $1 / 2$, and p38 play an important role in osteogenic differentiation (Ba et al. 2017). Knockdown of lncRNA DANCR can activate p38 MAPK to induce MSCs to differentiate into osteoblasts (Zhang et al. 2018). In addition, lncRNA SNHG1 can inhibit the activation of p38, thereby inhibiting the osteogenic differentiation of bone marrow mesenchymal stem cells (BMSCs) (Jiang et al. 2019).

Osteoclasts are derived from hematopoietic stem cells or mononuclear macrophages. They can differentiate into osteoclast precursor cells and mature under the regulation of RANKL secreted by osteoblasts (Ono and Nakashima 2018). RANKL is a key cytokine that regulates the differentiation of osteoclasts. It combines with the signal receptor RANK to promote the formation of osteoclasts. The osteoprotegerin (OPG) secreted by osteoblasts inhibits the combination of RANKL and RANK and inhibits the formation of osteoclasts. RANK/ RANKL/OPG is the main regulatory system for osteoclast differentiation, maturation, and survival (Udagawa et al. 2021). When osteoclast activity increases, it causes increased bone resorption and bone destruction (Fig. 1).

There are few studies involving the role of lncRNA in osteoclast differentiation, but some researchers have found that lncRNA does play a role in regulating this process. Chen et al. found that lncRNA bone marrow associated non-coding RNA (Bmncr) could inhibit RANKL-induced osteoclast differentiation and inhibit the progression of osteoporosis (Chen et al. 2019). LncRNA Jak3 was also found to increase the expression of cathepsin K (CTSK) through Jak3-mediated activation of T-cells 1 (NFATC1). Jak3 plays a key role in the differentiation of osteoclasts through Jak3/NFATC1/CTSK (Lee et al. 2019). NFATC1 is an essential transcription factor in the process of osteoclastogenesis, and lncRNA AK077216 promotes the expression of NFATC1 by inhibiting the expression of NFAT interacting protein (NIP45), thereby promoting the differentiation of osteoclasts (Liu et al. 2019).

The functional mechanism of lncRNA allows it to have an important role in the occurrence and development of osteoporosis. For instance, lncRNA CASC11 is up-regulated in the postmenopausal osteoporosis and is associated with TNF- $\alpha$ (Yu et al. 2019). TNF- $\alpha$ can inhibit the activity of osteoblasts at certain stages of osteogenic differentiation, and stimulate the proliferation and differentiation of osteoclasts (Wang and He 2020). In addition, lncRNA GAS5 is up-regulated in osteoporosis and 


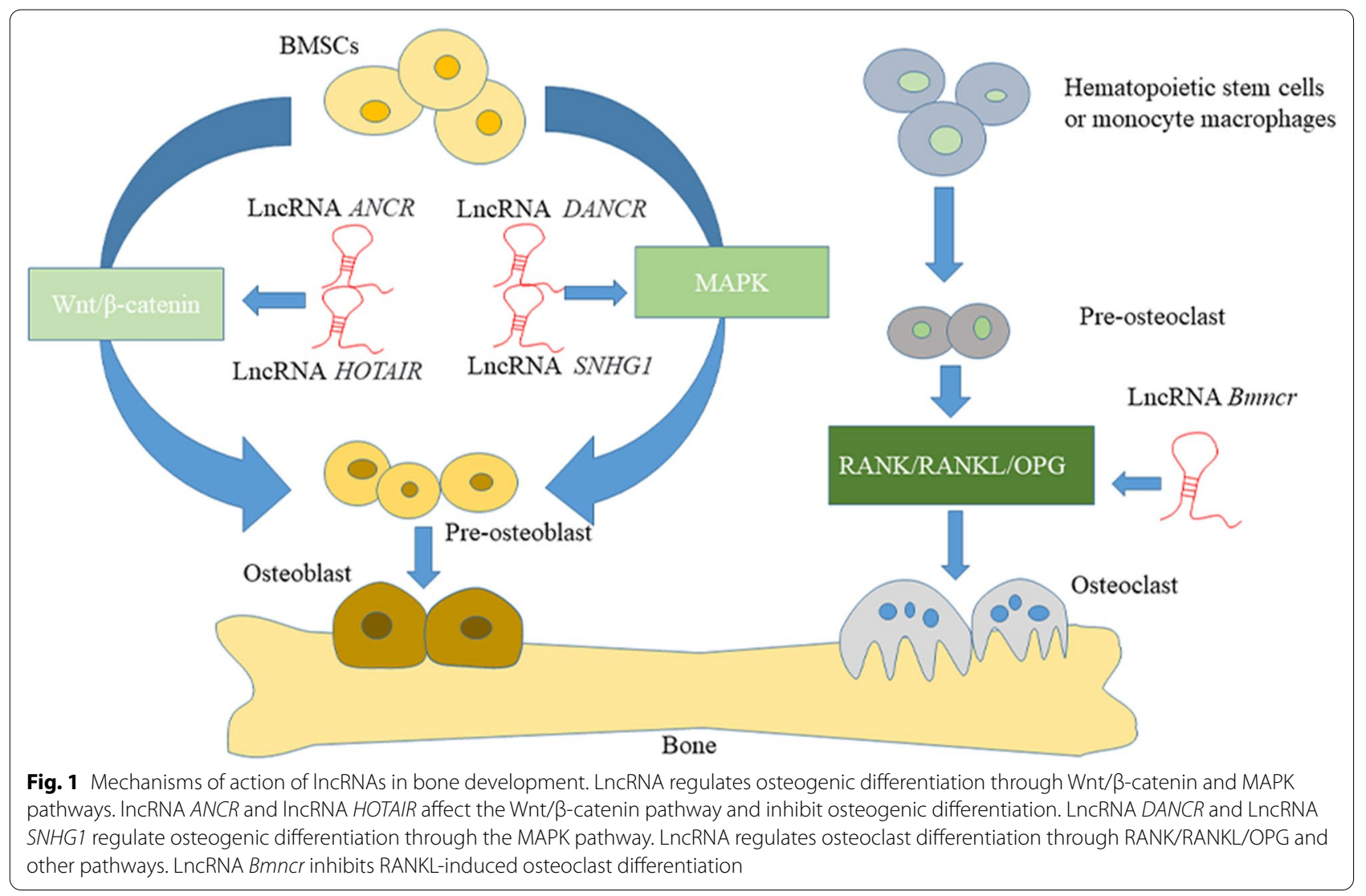

may down-regulate $m i R-21$ to promote osteoclast apoptosis (Cong et al. 2020). Further research is required to elucidate the role of abnormal lncRNA expression in the development mechanism of osteoporosis, as well as to discover the involved pathological effects and molecular mechanisms.

Through the increasing amount of research on this topic, many lncRNAs have been found to regulate important biological activities. Compared with coding RNA and miRNA, the research time involved with lncRNA is not as arduous; there are still many lncRNAs that have not yet been studied, and the function of these lncRNAs is still unclear. Researchers will be able to discover important gene regulatory functions faster and more effectively with the development of more high-throughput screening technologies such as microarray, the combination of a new generation of high-throughput sequencing technology, and using bioinformatics prediction tools. This will allow for more functional mechanisms of lncRNA to be discovered. Significantly, many lncRNAs are involved in bone remodeling and osteogenic differentiation, and they can influence the differentiation and activity of osteoblasts and osteoclasts. Despite the biological evidence, the specific mechanism of this process needs to be better understood through more research.

\section{LncRNA H19 in osteoporosis}

LncRNA H19 is located on chromosome 11p15.5 and is $2.3 \mathrm{~kb}$. It is only expressed in maternal alleles and plays an important role in regulating various biological functions, such as cancer and diabetes (Peng et al. 2018). The expression of $H 19$ is affected by many factors, including oncogenes. H19 has been confirmed to be up-regulated in many cancers and promotes the proliferation of cancer cells (Ghafouri-Fard et al. 2020). For example, up-regulation of $H 19$ and miR-675 promotes the proliferation of gastric cancer cells and inhibits apoptosis, while knocking down these two genes causes the opposite effect (Yan et al. 2017). In colorectal cancer cells, H19 mediates $m i R-138$ by sponging it and subsequently enhances the expression of HMGA1, thereby enhancing the invasion ability of cancer cells (Yang et al. 2017). H19 is one of the most conserved and abundant non-coding transcripts in mammalian development (Gabory et al. 2010) and has been proven to promote bone growth and differentiation (Dey et al. 2014). With the focused research on $H 19$, it has been found that $H 19$ can regulate osteoblast differentiation, mediate bone regeneration, and regulate bone metabolism. Based on the effects of H19 on the process of osteogenic differentiation, the potential use of H19 in the treatment of osteoporosis can be studied. In 
addition, $H 19$ has many connections to osteoporosis. Li et al. found that the up-regulation of DMNT1 can lead to the methylation of the $H 19$ promoter in osteoporotic rats and inhibit the ERK signaling pathway, which can cause disuse osteoporosis (Li et al. 2018). Further research is needed into how $H 19$ regulates osteogenic differentiation and how it participates in osteoporosis.

\section{Roles of IncRNA H19 in regulating osteogenic differentiation through modulation of the IncRNA-miRNA-mRNA network}

LncRNA $H 19$ can act as the upstream target gene of miRNAs, thereby regulating the expression of mRNA and influencing the process of osteogenic differentiation of osteoblasts. $\mathrm{H} 19$ is up-regulated during the process of osteogenic differentiation of MSCs, while knockdown of $H 19$ inhibits the proliferation of osteoblasts, both in vivo and in vitro. Huang et al. implanted hMSCs stably expressing H19, shH19-1 and control hMSCs into subcutaneous tissues of mice. Eight weeks later, it was observed that the bone formation of the H19-overexpressing group was higher than the control group, while the bone formation of the H19-knockdown group was reduced, which was consistent with the results of in vitro cell experiments (Huang et al. 2015). Studies have shown that miR675, which is inserted into the first exon of $H 19$, promotes the osteogenic differentiation of human mesenchymal stem cells. H19 is the precursor of miR675, and its first exon contains a hairpin consisting of miRNA. This hairpin was found to be a template of two different miRNAs (miR-675-5p and miR-675-3p) that can express miR-675 with 23 nucleotides (Cai and Cullen 2007; Keniry et al. 2012). In addition, $H 19$ forms self-regulating feedback with its encoded miR-675-5p. This indicates that miR-675-5p targets $H 19$ to form a negative regulatory loop. Both H19 and miR675 are up-regulated in osteoporosis differentiation and miR-675 down-regulates TGF- $\beta 1$ (Huang et al. 2015). Down-regulation of TGF- $\beta 1$ inhibits phosphorylation of Smad3 (Derynck and Zhang 2003), and it reduces the expression of histone deacetylase (HDAC4/5) required for osteogenic differentiation. Phosphorylation of Smad3 recruits HDAC4/5 to Runx2 and forms a stable complex on the DNA sequence bound by Runx2, thereby down-regulating the expression of Runx2 (Kang et al. 2005). The miR675 partially mediates the osteogenic activity of $H 19$, so the H19/miR-675/TGF$\beta 1 /$ Smad3/HDAC pathway can promote osteogenic differentiation, but further studies are needed to determine whether H19/miR-675 directly targets these factors or involves other mechanisms.

Bone morphogenetic proteins (BMPs) belong to the TGF- $\beta$ superfamily and play an important role in osteogenic differentiation and bone formation. BMP9 is the most significant type of BMPs that induces the osteogenic differentiation of MSCs. Interestingly, recent studies have found that $H 19$ can regulate downstream miRNAs (miR-107, miR-27b, miR-106b, miR-125a, and $m i R-17)$ during the process of osteogenic differentiation of MSCs to express ligands such as DII1, DII3, DII4, Jag1, and Jag2 (Liao et al. 2017). These ligands can regulate the Notch signaling pathway and promote BMP9 to induce osteogenic differentiation of MSCs, although the specific mechanism remains to be clarified. Studies have proved that activating the Notch signaling pathway through subcutaneous stem cell implantation and ectopic bone formation can restore the osteogenic deficiency of bone marrow mesenchymal stem cells caused by the knockdown of $H 19$, and cell experiments in vitro were consistent with the results (Liao et al. 2017).

In addition, knocking down $H 19$ reduces the expression of SATB2 by up-regulating $m i R-140-5 p$. The $m i R$ 140-5p inhibits the osteogenic differentiation of BMSCs by targeting SATB2. Therefore, H19 promotes the osteogenic differentiation of BMSCs by regulating the miR140-5p/SATB2 axis (Bi et al. 2020).

Previous studies have shown that $m i R-19 b-3 p$ is a positive regulator of osteogenic differentiation (Xiong et al. 2020). The mimic transfection of $m i R-19 b-3 p$ can significantly promote the protein expression levels of RUNX2 and COL1A1, while the transfection of the miR-19b-3p inhibitor can significantly inhibit the expression of these two proteins. However, overexpression of $H 19$ can down-regulate the expression of $m i R-19 b-3 p$ in BMSCs. Therefore, $H 19$ is down-regulated in postmenopausal osteoporosis and promotes the up-regulation of $m i R$ $19 b-3 p$. This controls the proliferation and osteogenic differentiation of BMSCs (Xiaoling et al. 2020).

\section{Roles of $\mathrm{H} 19$ as ceRNA to regulate osteogenic differentiation}

Recently, the ceRNA hypothesis proposed that lncRNA communicates with other protein-coding RNA transcripts by sharing a common miRNA binding site (Salmena et al. 2011). A large number of lncRNAs, including H19, are thought to play the role of a miRNA sponge (Liang et al. 2016). Through bioinformatics and genetic testing, Liang et al. found that $H 19$ is the miRNA sponge of miR141 and miR22, both of which are negative regulators of osteogenesis and the $\mathrm{Wnt} / \beta$ catenin pathway. H19 competitively binds to the miRNAs and antagonizes the functions of the two miRNAs. Their target genes $\beta$-catenin aggregate, thereby activating the $\mathrm{Wnt} / \beta$ catenin pathway to promote osteogenic differentiation (Liang et al. 2016).

$H 19$ can also act as the ceRNA of miR138, and then up-regulate the downstream FAK, and play a positive 
regulatory role in osteogenic differentiation. The specific mechanism involves miR-138 inhibiting PTK2 gene expression through competitively binding with $H 19$ and promoting FAK expression to induce osteogenic differentiation of MSCs (Wu et al. 2018a, b).

The $m i R-188$ is down-regulated in the osteogenic differentiation of mouse bone marrow mesenchymal stem cells (mBMSCs), while $H 19$ and LCoR are up-regulated in the adipogenic differentiation of mBMSCs (Wang et al. 2018). Knockdown of $H 19$ can significantly increase the expression of $m i R-188$. Therefore, as shown in Fig. 2, H19 can mediate LCoR and regulate the balance between osteogenic and adipogenic differentiation of mBMSCs through sponging miR-188.

\section{Roles of $H 19$ in regulating factors related to osteogenic differentiation}

H19 also participates in osteogenic differentiation by regulating some factors associated with osteogenic differentiation (Table 1). For instance, Li et al. constructed a disused osteoporosis model after mechanically unloading mice and detected low expression of $\mathrm{H} 19$ and high expression of DKK4 in these mice through gene sequencing (Li et al. 2017). The DKK family (DKK1-4) is an extracellular inhibitor of the $\mathrm{Wnt} / \beta$-catenin pathway and plays a negative role in the process of osteogenesis (Aslan et al. 2006; Fujita and Janz 2007; Hiramitsu et al. 2013). Bioinformatics analysis showed that DKK4 is the target gene of $H 19$, and through the $\mathrm{Wnt} / \beta$-catenin pathway to mediate the biological effects of $H 19$ in disused osteoporosis ( $\mathrm{Li}$ et al. 2017). Then, cell experiments were conducted to verify this, which resulted in the discovery that knockdown of $\mathrm{H} 19$ can inhibit the Wnt/ $\beta$-catenin pathway and osteogenic function. However, the knockdown of DKK4 can prevent this effect. Therefore, this result suggests that $H 19$ targets DKK4 to stimulate the $\mathrm{Wnt} / \beta$-catenin pathway and promotes osteogenesis to prevent disused osteoporosis (Li et al. 2017).

Forkhead box c2 (Foxc2) is a member of the wingedhelix/forkhead transcription factor family and participates in many biological processes. Overexpression of Foxc2 in bone marrow mesenchymal stem cells can promote osteogenic differentiation and inhibit adipogenic differentiation (You et al. 2013). At the same time, Foxc2 stimulates osteoblast differentiation of mesenchymal cells by activating the Wnt/ $\beta$-catenin pathway (Kim et al.

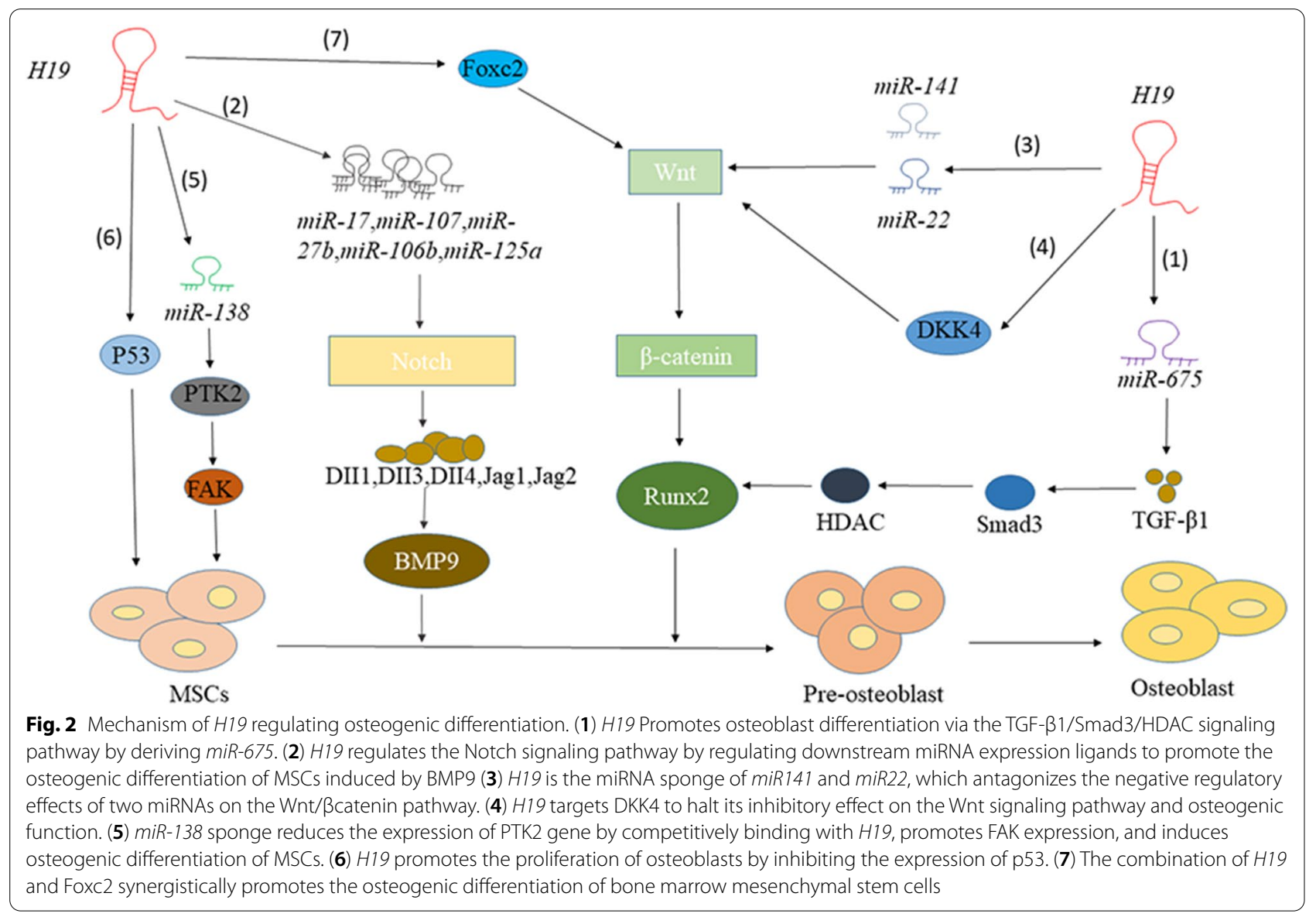


Table.1 Different roles of H19 in promoting osteogenic differentiation

\begin{tabular}{|c|c|c|c|c|c|}
\hline Expression of $H 19$ & Sampling tissue & Mode of action & $\begin{array}{l}\text { Targets and pathways } \\
\text { affected }\end{array}$ & Changes of targets & Refs. \\
\hline Up-regulated & Human BMSCs & Precursor of miR- 675 & $\begin{array}{l}\text { miR- } 675 / T G F-\beta 1 / S m a d 3 / \\
\text { HDAC axis } \\
\text { Promoting Wnt/ } \beta- \\
\text { catenin pathway }\end{array}$ & Up-regulated of miR-675 & Huang et al. (2015) \\
\hline Up-regulated & Mouse BMSCs & $\begin{array}{l}\text { Regulating miRNAs that } \\
\text { target Notch ligands and } \\
\text { receptors }\end{array}$ & $\begin{array}{l}\text { miRNAs/ligands/Notch/ } \\
\text { BMP9 axis } \\
\text { Promoting Notch } \\
\text { pathway }\end{array}$ & Up-regulated of miRNAs & Liao et al. (2017) \\
\hline Up-regulated & $\begin{array}{l}\text { Human serum and } \\
\text { Mouse BMSCs }\end{array}$ & Binding to FOX2 & $\begin{array}{l}\text { FOX2 } \\
\text { Promoting Wnt/ß- } \\
\text { catenin pathway }\end{array}$ & Up-regulated & Zhou et al. (2019) \\
\hline Up-regulated & Femurs of rats & Targeting DKK4 & $\begin{array}{l}\text { DKK4 } \\
\text { Promoting Wnt/ß- } \\
\text { catenin pathway }\end{array}$ & Down-regulated & Li et al. (2017) \\
\hline Down-regulated & $\begin{array}{l}\text { Human blood and } \\
\text { Human BMSCs }\end{array}$ & Mediating miR-19b-3p & $\begin{array}{l}\text { miR-19b-3p } \\
\text { H19/miR-19b-3p/RUNX2 } \\
\text { axis }\end{array}$ & Up-regulated & Xiaoling et al. (2020) \\
\hline Up-regulated & Tibia of mice & Binding to P53 & P53 & Down-regulated & Zhou et al. (2018) \\
\hline Up-regulated & Human BMSCs & Sponging for miR-138 & $\begin{array}{l}\text { miR-138/FAK/ERK/Runx2 } \\
\text { axis }\end{array}$ & Up-regulated & Wu et al. $(2018 a, b)$ \\
\hline Up-regulated & Human BMSCs & $\begin{array}{l}\text { Sponging for miR-141 } \\
\text { and miR-22 }\end{array}$ & $\begin{array}{l}\text { miR-141 and miR-22 } \\
\text { Promoting Wnt/ } \beta- \\
\text { catenin pathway }\end{array}$ & Up-regulated & Liang et al. (2016) \\
\hline Up-regulated & Mouse BMSCs & Sponging for miR-188 & H19/miR-188/LCoR axis & Down-regulated & Wang et al. (2018) \\
\hline Up-regulated & Human BMSCs & Sponging for miR-140-5p & $\begin{array}{l}\text { H19miR-140-5p/SATB2 } \\
\text { axis }\end{array}$ & $\begin{array}{l}\text { Down-regulated of miR- } \\
140-5 p\end{array}$ & Bi et al. (2020) \\
\hline Up-regulated & Human BMSCs & Sponging for miR-214-5p & $\begin{array}{l}\text { H19/miR-214-5p/BMP2 } \\
\text { axis }\end{array}$ & $\begin{array}{l}\text { Down-regulated of miR- } \\
214-5 p\end{array}$ & He et al. (2021) \\
\hline
\end{tabular}

BMSCs bone marrow mesenchymal cells, miRNAs microRNAs, HADC Histone deacetylase, BMPs bone morphogenetic proteins, FOXC2 Forkhead box c2, DKK4 Dickkopf protein 4, FAK Focal adhesion kinase, ERK extracellular signal-regulated kinase, LCOR Ligand-dependent corepressor, SATB2 special AT-rich sequence-binding protein 2

2009). Foxc2 can promote the transcription of Wnt4 promoter by combining with the Wnt4 promoter, and the overexpression of Foxc2 in BMSCs can promote osteogenic differentiation through the Wnt/ $\beta$-catenin pathway. The overexpression of $H 19$ can increase the expression of Wnt4, and the Wnt4 luciferase report shows that the activity of the Wnt- $\beta /$ catenin pathway is enhanced after Foxc 2 co-transfection with $H 19$ (Zhou et al. 2019). Therefore, the combination of $H 19$ and Foxc 2 can promote the osteogenic differentiation of bone marrow mesenchymal stem cells through the $\mathrm{Wnt} / \beta$-catenin pathway.

In addition to these pathways and regulatory mechanisms, research on how $H 19$ regulates osteogenic differentiation is gradually increasing. Zhou et al. established a tibial fracture model in mice to detect the expression levels of H19 and p53 (Zhou et al. 2018). The experimental results show that in the process of osteogenic differentiation of MSCs, $H 19$ directly binds to p53 and inhibits the activity of p53 to promote the osteogenic differentiation of MSCs. Therefore, H19, as a molecular marker to promote fracture healing, induces the proliferation of bone cells by inhibiting the expression of p53.

\section{Roles of $\mathrm{H} 19$ in regulating osteoclasts}

The important role of IncRNA in osteoporosis has attracted the attention of researchers. However, there is little research on the role of $\mathrm{H} 19$ in the proliferation and differentiation of osteoclasts. Some studies have shown that miR-29a-3p is the target of H19, and the expression of $H 19$ is up-regulated in patients with osteoporosis (Li et al. 2020). The overexpression of $H 19$ can promote the expression of inflammatory mediators such as TNF$\alpha$, IL-1 $\beta$, and IL- 10 by osteoclasts. After the knockdown of $H 19$, the promotion effect is inhibited, and the proliferation of osteoclasts is also inhibited. Additionally, after co-transfection with the $m i R-29 a-3 p$ inhibitor and siRNA-H19, $H 19$ has a weakened regulatory effect on the expression of pro-inflammatory mediators, cell proliferation, and apoptosis. Therefore, the H19/miR-29a-3p axis is also involved in the development of osteoporosis by regulating osteoclasts ( $\mathrm{Li}$ et al. 2020). Furthermore, there are some studies on $\mathrm{H} 19$ in the blood of patients with osteoporosis. According to the latest research, H19 is down-regulated in the plasma of postmenopausal patients with osteoporosis and can control osteogenic 
differentiation by targeting related factors to participate in osteoporosis. This is consistent with the results of previous studies (Xiaoling et al. 2020).

\section{DNA methylation of $\mathrm{H} 19$ in the osteogenic differentiation}

Currently, epigenetics is developing rapidly, and DNA methylation regulation is one of the key components being studied (Meng et al. 2015). Two genetic imprinting genes, IGF2/H19, are located on the $11 \mathrm{p} 155$ chromosomal regions. IGF2 is a maternally imprinted gene and is expressed by the father, while H19 is paternal and expressed by the mother (Nordin et al. 2014). H19 and IGF2 are expressed by opposite parental alleles but are jointly regulated. They share a common imprinting mechanism and are downregulated in many human cancers and fetal growth syndromes. A study found that when the methylation defect occurred in this region, it led to Beckwith-Wiedemann syndrome (BWS) (Fontana et al. 2021). The combination of H19 and S-adenosylhomocysteine hydrolase (SAHH) can interfere with the hydrolysis ability of S-adenosylhomocysteine (SAH), and SAH is an effective product inhibitor of adenylatedependent methyltransferase. This process leads to defective methylation of the targeted gene, which affects its activity (Zhou et al. 2015). In addition, the osteogenic activity in the aortic valve is controlled by $\mathrm{NOTCH} 1$, one of the members of the Notch family, which regulates the expression of key osteogenic genes, such as RUNX2 and BMP2. During calcific aortic valve disease, DNA methylation in the $H 19$ promoter is dysregulated, causing $H 19$ to interfere with the expression of NOTCH1 to promote the osteogenesis process in the aortic valve (Hadji et al. 2016). This evidence proves that $H 19$ can affect osteogenic differentiation through DNA methylation. The research on the relationship between the methylation status of gene imprinting and osteoporosis will be an emerging research direction in the field of bone metabolism. An in-depth understanding of the DNA methylation mechanism of $H 19$ will provide a new direction for the treatment of osteoporosis.

\section{Conclusion}

In this review, we have been elaborating on the common functions of IncRNA, focusing on summarizing the role of $H 19$ in osteogenic differentiation and how it participates in osteoporotic activities. The roles of $H 19$ in osteogenic differentiation can be described in three ways: LncRNA H19 directly participates in osteogenesis through the IncRNA-miRNA-mRNA network. For instance, $H 19$ promotes osteogenic differentiation through the H19/miR-675/TGF- $\beta 1 / \mathrm{Smad} 3 / \mathrm{HDAC}$ pathway (Huang et al. 2015). Another role of H19 is to indirectly participate in osteogenesis through the action of ceRNA, by absorbing miRNAs and competing with miRNA to regulate the encoded protein. Finally, lncRNA H19 regulates osteogenic differentiation by influencing certain factors. In addition, studies have found that H19 mediates estrogen-regulated osteogenic differentiation in BMSCs through the miR-532-3p/SIRT1 axis, and estrogen can mediate $m i R-532-3 p /$ SIRT1 axis to reduce osteoporosis in ovariectomy rats by up-regulating $H 19$ (Li et al. 2021). These findings provide new ideas for the incidence and development of osteoporosis and provide new targets for its prevention and treatment. However, the regulation mechanism of $H 19$ has not been fully elucidated, and the effect of $H 19$ on downstream molecules is still unknown. The clinical application of $H 19$ and its use in the treatment of bone metabolism diseases are key topics for future studies.

\begin{abstract}
Abbreviations
IncRNAs: Long non-coding RNAs; miRNAs: MicroRNAs; ceRNAs: Competing endogenous RNAs; MSCs: Mesenchymal stem cells; BMSCs: Bone marrow mesenchymal stem cells; hBMSCs: Human Bone marrow mesenchymal stem cells; mBMSCs: Mouse Bone marrow mesenchymal stem cells; Bmncr: Bone marrow associated non-coding RNA; HDAC: Histone deacetylase; LCoR: Liganddependent corepressor; NF-KB: Nuclear factor-KB; MAPK: Mitogen-activated protein kinase; siRNA: Small interfering RNA; mRNA: Messenger RNA; ANCR: Anti-differentiation noncoding RNA; HOTAIR: HOX transcript antisense RNA; JNK: C-Jun amino terminal kinase; ERK: Extracellular signal-regulated kinase; SNHG1: Small nucleolar RNA host gene; RANK: Receptor activator of NFkappaB; RANKL: Receptor activator of NF-kappaB ligand; CASC11: Cancer susceptibility 11; TNF: Transforming growth factor; GAS5: Growth arrest-specific transcript 5; HMGA1: High mobility group A1; DMNT1: DNA methyltransferase 1; DKK4: Dickkopf protein 4; Foxc2: Forkhead box c2; BMPs: Bone morphogenetic proteins; DII: Dietary inflammatory index; Jag: Jagged; SATB2: Special AT-rich sequence-binding protein 2; FAK: Focal adhesion kinase; PTK: Protein tyrosine kinases; SIRT1: Silencing information regulator 2 related enzyme 1; SAHH: S-adenosylhomocysteine hydrolase; SAH: S-adenosylhomocysteine.
\end{abstract}

\section{Acknowledgements}

Not applicable.

\section{Authors' contributions}

SC researched the literature and drafted the manuscript. ZZ and SQ revised the manuscript. DL revised the manuscript and approved the final version. All authors read and approved the final manuscript.

\section{Funding}

This work was supported by grants from the National Natural Science Foundation of China [Grant No. 81972156], the Natural Science Foundation of Liaoning Province [Grant No. 2019-ZD-0781] and 345 Talent Project.

Availability of data and materials

Not applicable.

\section{Declarations}

Ethics approval and consent to participate

Not applicable.

Consent for publication

Not applicable.

Competing interests

The authors declare that they have no competing interests. 
Received: 25 March 2021 Accepted: 22 September 2021

Published online: 28 September 2021

\section{References}

Aslan H, Ravid-Amir O, Clancy BM, Rezvankhah S, Pittman D, Pelled G, Turgeman G, Zilberman Y, Gazit Z, Hoffmann A, et al. Advanced molecular profiling in vivo detects novel function of dickkopf-3 in the regulation of bone formation. J Bone Miner Res. 2006;21:1935-45.

Ba P, Duan X, Fu G, Lv S, Yang P, Sun Q. Differential effects of p38 and Erk1/2 on the chondrogenic and osteogenic differentiation of dental pulp stem cells. Mol Med Rep. 2017;16:63-8.

Bi HU, Wang D, Liu X, Wang G, Wu X. Long non-coding RNA H19 promotes osteogenic differentiation of human bone marrow-derived mesenchymal stem cells by regulating microRNA-140-5p/SATB2 axis. J Biosci. 2020;45:56.

Cai X, Cullen BR. The imprinted $\mathrm{H} 19$ noncoding RNA is a primary microRNA precursor. RNA. 2007;13:313-6.

Chan JJ, Tay Y. Noncoding RNA: RNA regulatory networks in cancer. Int J Mol Sci. 2018;19(5):1310.

Chen LL. Linking long noncoding RNA localization and function. Trends Biochem Sci. 2016;41:761-72.

Chen $\mathrm{H}$, Senda T, Kubo KY. The osteocyte plays multiple roles in bone remodeling and mineral homeostasis. Med Mol Morphol. 2015;48:61-8.

Chen RS, Zhang XB, Zhu XT, Wang CS. LncRNA Bmncr alleviates the progression of osteoporosis by inhibiting RANML-induced osteoclast differentiation. Eur Rev Med Pharmacol Sci. 2019;23:9199-206.

Cong C, Tian J, Gao T, Zhou C, Wang Y, Cui X, Zhu L. IncRNA GAS5 is upregulated in osteoporosis and downregulates miR-21 to promote apoptosis of osteoclasts. Clin Interv Aging. 2020;15:1163-9.

Derynck R, Zhang YE. Smad-dependent and Smad-independent pathways in TGF-beta family signalling. Nature. 2003;425:577-84.

Dey BK, Pfeifer K, Dutta A. The H19 long noncoding RNA gives rise to microRNAs miR-675-3p and miR-675-5p to promote skeletal muscle differentiation and regeneration. Genes Dev. 2014;28:491-501.

Dykes IM, Emanueli C. Transcriptional and post-transcriptional gene regulation by long non-coding RNA. Genomics Proteomics Bioinformatics. 2017;15:177-86

Fang Y, Fullwood MJ. Roles, functions, and mechanisms of long non-coding RNAs in cancer. Genomics Proteomics Bioinformatics. 2016;14:42-54.

Fontana L, Tabano S, Maitz S, Colapietro P, Garzia E, Gerli AG, Sirchia SM, Miozzo M. Clinical and molecular diagnosis of beckwith-wiedemann syndrome with single- or multi-locus imprinting disturbance. Int J Mol Sci. 2021;22(7):3445.

Fujita K, Janz S. Attenuation of WNT signaling by DKK-1 and -2 regulates BMP2induced osteoblast differentiation and expression of OPG RANKL and M-CSF. Mol Cancer. 2007;6:71.

Gabory A, Jammes H, Dandolo L. The H19 locus: role of an imprinted noncoding RNA in growth and development. BioEssays. 2010;32:473-80.

Ghafouri-Fard S, Esmaeili M, Taheri M. H19 IncRNA: Roles in tumorigenesis. Biomed Pharmacother. 2020;123:109774.

Gibbons HR, Shaginurova G, Kim LC, Chapman N, Spurlock CF 3rd, Aune TM. Divergent IncRNA GATA3-AS1 regulates GATA3 transcription in T-Helper 2 cells. Front Immunol. 2018;9:2512.

Hadji F, Boulanger MC, Guay SP, Gaudreault N, Amellah S, Mkannez G, Bouchareb R, Marchand JT, Nsaibia MJ, Guauque-Olarte S, et al. Altered DNA methylation of long noncoding RNA H19 in calcific aortic valve disease promotes mineralization by silencing NOTCH1. Circulation. 2016;134:1848-62.

Harvey N, Dennison E, Cooper C. Osteoporosis: impact on health and economics. Nat Rev Rheumatol. 2010;6:99-105.

Hassan MQ, Tye CE, Stein GS, Lian JB. Non-coding RNAs: Epigenetic regulators of bone development and homeostasis. Bone. 2015;81:746-56.

He Q, Li R, Hu B, Li X, Wu Y, Sun P, Jia Y, Guo Y. Stromal cell-derived factor-1 promotes osteoblastic differentiation of human bone marrow mesenchymal stem cells via the IncRNA-H19/miR-214-5p/BMP2 axis. J Gene Med. 2021. https://doi.org/10.1002/jgm.3366.

Hernlund E, Svedbom A, Ivergård M, Compston J, Cooper C, Stenmark J, McCloskey EV, Jönsson B, Kanis JA. Osteoporosis in the European Union: medical management, epidemiology and economic burden. A report prepared in collaboration with the International Osteoporosis Foundation (IOF) and the European Federation of Pharmaceutical Industry Associations (EFPIA). Arch Osteoporos. 2013;8:136.

Hiramitsu S, Terauchi M, Kubota T. The effects of Dickkopf-4 on the proliferation, differentiation, and apoptosis of osteoblasts. Endocrinology. 2013;154:4618-26.

Huang Y, Zheng Y, Jia L, Li W. Long noncoding RNA H19 promotes osteoblast differentiation via TGF- $\beta 1 /$ Smad3/HDAC signaling pathway by deriving miR-675. Stem Cells. 2015;33:3481-92.

Jathar S, Kumar V, Srivastava J, Tripathi V. Technological developments in IncRNA biology. Adv Exp Med Biol. 2017;1008:283-323.

Jia Q, Jiang W, Ni L. Down-regulated non-coding RNA (IncRNA-ANCR) promotes osteogenic differentiation of periodontal ligament stem cells. Arch Oral Biol. 2015;60:234-41.

Jiang Y, Wu W, Jiao G, Chen Y, Liu H. LncRNA SNHG1 modulates p38 MAPK pathway through Nedd 4 and thus inhibits osteogenic differentiation of bone marrow mesenchymal stem cells. Life Sci. 2019;228:208-14.

Johnston CB, Dagar M. Osteoporosis in Older Adults. Med Clin North Am. 2020;104:873-84

Kang JS, Alliston T, Delston R, Derynck R. Repression of Runx2 function by TGF-beta through recruitment of class II histone deacetylases by Smad3. Embo j. 2005;24:2543-55.

Keniry A, Oxley D, Monnier P, Kyba M, Dandolo L, Smits G, Reik W. The H19 lincRNA is a developmental reservoir of miR-675 that suppresses growth and Igf1r. Nat Cell Biol. 2012;14:659-65.

Khan M, Cheung AM, Khan AA. Drug-related adverse events of osteoporosis therapy. Endocrinol Metab Clin North Am. 2017;46:181-92.

Kim ED, Sung S. Long noncoding RNA: unveiling hidden layer of gene regulatory networks. Trends Plant Sci. 2012;17:16-21.

Kim SH, Cho KW, Choi HS, Park SJ, Rhee Y, Jung HS, Lim SK. The forkhead transcription factor Foxc2 stimulates osteoblast differentiation. Biochem Biophys Res Commun. 2009;386:532-6.

Kopp F, Mendell JT. Functional classification and experimental dissection of long noncoding RNAs. Cell. 2018;172:393-407.

Krol J, Krol I, Alvarez CP, Fiscella M, Hierlemann A, Roska B, Filipowicz W. A network comprising short and long noncoding RNAs and RNA helicase controls mouse retina architecture. Nat Commun. 2015;6:7305.

Lee CP, Huang YN, Nithiyanantham S, Huang CM, Ko YC. LnCRNA-Jak3:Jak3 coexpressed pattern regulates monosodium urate crystal-induced osteoclast differentiation through Nfatc1/Ctsk expression. Environ Toxicol. 2019;34:179-87.

Li B, Liu J, Zhao J, Ma JX, Jia HB, Zhang Y, Xing GS, Ma XL. LncRNA-H19 modulates Wnt/ $\beta$-catenin signaling by targeting Dkk4 in hindlimb unloaded rat. Orthop Surg. 2017:9:319-27.

Li B, Zhao J, Ma JX, Li GM, Zhang Y, Xing GS, Liu J, Ma XL. Overexpression of DNMT1 leads to hypermethylation of $\mathrm{H} 19$ promoter and inhibition of Erk signaling pathway in disuse osteoporosis. Bone. 2018;111:82-91.

Li Z, Hong Z, Zheng Y, Dong Y, He W, Yuan Y, Guo J. An emerging potential therapeutic target for osteoporosis: LncRNA H19/miR-29a-3p axis. Eur J Histochem. 2020;64(4):3155

Li J, Wang X, Wang Y, Yang Q. H19 promotes the gastric carcinogenesis by sponging miR-29a-3p: evidence from IncRNA-miRNA-mRNA network analysis. Epigenomics. 2020;12:989-1002.

Li T, Jiang H, Li Y, Zhao X, Ding H. Estrogen promotes IncRNA H19 expression to regulate osteogenic differentiation of BMSCs and reduce osteoporosis via miR-532-3p/SIRT1 axis. Mol Cell Endocrinol. 2021;527:111171.

Liang WC, Fu WM, Wang YB, Sun YX, Xu LL, Wong CW, Chan KM, Li G, Waye MM, Zhang JF. H19 activates Wnt signaling and promotes osteoblast differentiation by functioning as a competing endogenous RNA. Sci Rep. 2016;6:20121.

Liao J, Yu X, Hu X, Fan J, Wang J, Zhang Z, Zhao C, Zeng Z, Shu Y, Zhang R, et al. IncRNA H19 mediates BMP9-induced osteogenic differentiation of mesenchymal stem cells (MSCs) through Notch signaling. Oncotarget. 2017:8:53581-601.

Liu C, Cao Z, Bai Y, Dou C, Gong X, Liang M, Dong R, Quan H, Li J, Dai J, et al. LncRNA AK077216 promotes RANKL-induced osteoclastogenesis and bone resorption via NFATC1 by inhibition of NIP45. J Cell Physiol. 2019;234:1606-17.

Ma L, Bajic VB, Zhang Z. On the classification of long non-coding RNAs. RNA Biol. 2013;10:925-33. 
Majidinia M, Sadeghpour A, Yousefi B. The roles of signaling pathways in bone repair and regeneration. J Cell Physiol. 2018;233:2937-48.

Meng H, Cao Y, Qin J, Song X, Zhang Q, Shi Y, Cao L. DNA methylation, its mediators and genome integrity. Int J Biol Sci. 2015;11:604-17.

Noh JY, Yang Y, Jung H. Molecular mechanisms and emerging therapeutics for osteoporosis. Int J Mol Sci. 2020;21(20):7623.

Nordin M, Bergman D, Halje M, Engström W, Ward A. Epigenetic regulation of the Igf2/H19 gene cluster. Cell Prolif. 2014;47:189-99.

Nusse R. Wnt signaling. Cold Spring Harb Perspect Biol. 2012. https://doi.org/ 10.1101/cshperspect.a011163.

Ono T, Nakashima T. Recent advances in osteoclast biology. Histochem Cell Biol. 2018;149:325-41.

Peng S, Cao L, He S, Zhong Y, Ma H, Zhang Y, Shuai C. An overview of long noncoding RNAs involved in bone regeneration from mesenchymal stem cells. Stem Cells Int. 2018;2018:8273648.

Ponting CP, Oliver PL, Reik W. Evolution and functions of long noncoding RNAs. Cell. 2009;136:629-41.

Pu M, Chen J, Tao Z, Miao L, Qi X, Wang Y, Ren J. Regulatory network of miRNA on its target: coordination between transcriptional and post-transcriptional regulation of gene expression. Cell Mol Life Sci. 2019;76:441-51.

Rachner TD, Khosla S, Hofbauer LC. Osteoporosis: now and the future. Lancet. 2011;377:1276-87.

Ransohoff JD, Wei Y, Khavari PA. The functions and unique features of long intergenic non-coding RNA. Nat Rev Mol Cell Biol. 2018;19:143-57.

Rion N, Rüegg MA. LncRNA-encoded peptides: more than translational noise? Cell Res. 2017;27:604-5.

Salmena L, Poliseno L, Tay Y, Kats L, Pandolfi PP. A ceRNA hypothesis: the Rosetta Stone of a hidden RNA language? Cell. 2011;146:353-8.

Schmitz SU, Grote P, Herrmann BG. Mechanisms of long noncoding RNA function in development and disease. Cell Mol Life Sci. 2016;73:2491-509.

Shen JJ, Zhang CH, Chen ZW, Wang ZX, Yang DC, Zhang FL, Feng KH. LncRNA HOTAIR inhibited osteogenic differentiation of BMSCs by regulating Wnt/ß-catenin pathway. Eur Rev Med Pharmacol Sci. 2019;23:7232-46.

Sun Y, Zhu Q, Yang W, Shan Y, Yu Z, Zhang Q, Wu H. LncRNA H19/miR-194/ PFTK1 axis modulates the cell proliferation and migration of pancreatic cancer. J Cell Biochem. 2019;120:3874-86.

Trajanoska K, Rivadeneira F. The genetic architecture of osteoporosis and fracture risk. Bone. 2019;126:2-10.

Udagawa N, Koide M, Nakamura M, Nakamichi Y, Yamashita T, Uehara S, Kobayashi Y, Furuya Y, Yasuda H, Fukuda C, et al. Osteoclast differentiation by RANKL and OPG signaling pathways. J Bone Miner Metab. 2021;39:19-26.

van Wijnen AJ, Westendorf JJ. Epigenetics as a new frontier in orthopedic regenerative medicine and oncology. J Orthop Res. 2019;37:1465-74.

Wang KC, Chang HY. Molecular mechanisms of long noncoding RNAs. Mol Cell. 2011;43:904-14.

Wang T, He C. TNF-a and IL-6: the link between immune and bone system. Curr Drug Targets. 2020;21:213-27.

Wang Y, Liu W, Liu Y, Cui J, Zhao Z, Cao H, Fu Z, Liu B. Long noncoding RNA H19 mediates LCoR to impact the osteogenic and adipogenic differentiation of mBMSCs in mice through sponging miR-188. J Cell Physiol. 2018;233:7435-46.

Wang R, Li Y, Du P, Zhang X, Li X, Cheng G. Hypomethylation of the IncRNA SOX21-AS1 has clinical prognostic value in cervical cancer. Life Sci. 2019;233:116708.

Wei JW, Huang K, Yang C, Kang CS. Non-coding RNAs as regulators in epigenetics (Review). Oncol Rep. 2017;37:3-9.

Wu P, Zuo X, Deng H, Liu X, Liu L, Ji A. Roles of long noncoding RNAs in brain development, functional diversification and neurodegenerative diseases. Brain Res Bull. 2013;97:69-80.
Wu J, Zhao J, Sun L, Pan Y, Wang H, Zhang WB. Long non-coding RNA H19 mediates mechanical tension-induced osteogenesis of bone marrow mesenchymal stem cells via FAK by sponging miR-138. Bone. 2018a;108:62-70.

Wu QY, Li X, Miao ZN, Ye JX, Wang B, Zhang F, Xu RS, Jiang DL, Zhao MD, Yuan FL. Long non-coding RNAs: a new regulatory code for osteoporosis. Front Endocrinol (lausanne). 2018b;9:587.

Xiaoling G, Shuaibin L, Kailu L. MicroRNA-19b-3p promotes cell proliferation and osteogenic differentiation of BMSCs by interacting with IncRNA H19. BMC Med Genet. 2020;21:11.

Xiong A, He Y, Gao L, Li G, Weng J, Kang B, Wang D, Zeng H. Smurf1-targeting miR-19b-3p-modified BMSCs combined PLLA composite scaffold to enhance osteogenic activity and treat critical-sized bone defects. Biomater Sci. 2020;8:6069-81.

Yan J, Zhang Y, She Q, Li X, Peng L, Wang X, Liu S, Shen X, Zhang W, Dong Y, et al. Long noncoding RNA H19/miR-675 axis promotes gastric cancer via FADD/Caspase 8/Caspase 3 signaling pathway. Cell Physiol Biochem. 2017:42:2364-76.

Yang Q, Wang X, Tang C, Chen X, He J. H19 promotes the migration and invasion of colon cancer by sponging miR-138 to upregulate the expression of HMGA1. Int J Oncol. 2017;50:1801-9.

Yang TL, Shen H, Liu A, Dong SS, Zhang L, Deng FY, Zhao Q, Deng HW. A road map for understanding molecular and genetic determinants of osteoporosis. Nat Rev Endocrinol. 2020;16:91-103.

You W, Gao H, Fan L, Duan D, Wang C, Wang K. Foxc2 regulates osteogenesis and angiogenesis of bone marrow mesenchymal stem cells. BMC Musculoskelet Disord. 2013;14:199.

Yu H, Zhou W, Yan W, Xu Z, Xie Y, Zhang P. LnCRNA CASC11 is upregulated in postmenopausal osteoporosis and is correlated with TNF-a. Clin Interv Aging. 2019;14:1663-9.

Zhang J, Tao Z, Wang Y. Long non-coding RNA DANCR regulates the proliferation and osteogenic differentiation of human bone-derived marrow mesenchymal stem cells via the p38 MAPK pathway. Int J Mol Med. 2018;41:213-9.

Zheng JF, Guo NH, Zi FM, Cheng J. Long noncoding RNA H19 promotes tumorigenesis of multiple myeloma by activating BRD4 signaling by targeting microRNA 152-3p. Mol Cell Biol. 2020;40(3):e00382-e419.

Zhou J, Yang L, Zhong T, Mueller M, Men Y, Zhang N, Xie J, Giang K, Chung H, Sun $\mathrm{X}$, et al. H19 IncRNA alters DNA methylation genome wide by regulating S-adenosylhomocysteine hydrolase. Nat Commun. 2015;6:10221.

Zhou QP, Zhang F, Zhang J, Ma D. H19 promotes the proliferation of osteocytes by inhibiting p53 during fracture healing. Eur Rev Med Pharmacol Sci. 2018;22:2226-32.

Zhou P, Li Y, Di R, Yang Y, Meng S, Song F, Ma L. H19 and Foxc2 synergistically promotes osteogenic differentiation of BMSCs via Wnt- $\beta$-catenin pathway. J Cell Physiol. 2019;234:13799-806.

Zhou Z, Hossain MS, Liu D. Involvement of the long noncoding RNA H19 in osteogenic differentiation and bone regeneration. Stem Cell Res Ther. 2021;12:74.

Zou Z, Liu W, Cao L, Liu Y, He T, Peng S, Shuai C. Advances in the occurrence and biotherapy of osteoporosis. Biochem Soc Trans. 2020;48:1623-36.

\section{Publisher's Note}

Springer Nature remains neutral with regard to jurisdictional claims in published maps and institutional affiliations. 